\title{
Platelet-rich plasma. Evidenze, limiti e prospettive future
}

\author{
A. Bove ${ }^{a}(\bowtie)$, B. Russo, F. Traficante, A. Barile, N. Orabona \\ U.O.C. di Ortopedia e Traumatologia, Ospedale del Mare, ASL Napoli 1 Centro, Napoli, Italia \\ above75@libero.it
}

\begin{abstract}
Platelet-rich Plasma. Evidence, limits and fUture Perspectives
Regenerative medicine is a promising frontier in several fields of medicine and surgery. In orthopaedics, cell products are used to facilitate the regeneration of bone, tendons, and cartilage in many orthopaedic disorders. A large portion of scientific literature is developing while basic science provides further information on tissue healing and the biological pathways to enhance it. PRP is one of the most promising products used as biological therapy. Even though a standardised methodology of preparation and utilisation is still missing, promising results coming from clinical research encourage its use. Further research will meet the challenge of standardising procedures, timing and way of application, and outcomes reporting, so as to allow researcher to make analyses and comparisons that will expand our knowledge of this interesting field of medicine.
\end{abstract}

Pubblicato online: 16 settembre 2019

(C) The Author(s) 2019

\section{Introduzione}

Negli ultimi anni si è registrato un interesse sempre crescente verso la Medicina Rigenerativa, quella branca della medicina, di sviluppo relativamente recente, che si occupa della riparazione, rigenerazione e sostituzione di tessuti e organi e del ripristino delle funzioni compromesse da infortuni, patologie e invecchiamento.

Sull'onda di questo interesse si sono moltiplicati gli studi condotti in vitro sulla rigenerazione tissutale e la loro applicazione in differenti aree cliniche e, sebbene ci siano stati inevitabili fallimenti iniziali, al momento i risultati positivi cominciano a diventare sempre più consistenti. Notevole interesse hanno fatto registrare le possibilità di applicazione di terapie biologiche per il trattamento delle patologie degenerative in ambito ortopedico, in virtù del potenziale di accelerare i processi di guarigione attraverso meccanismi di rigenerazione o immunomodulazione [1]. Numerosi e differenti approcci biologici sono stati proposti per queste patologie, fra cui il Platelet Rich Plasma (PRP), il Bone Marrow Aspirate Concentrate (BMAC) e le terapie cellulari.

Nonostante i numerosi sforzi della ricerca $[2,3]$, tuttavia, non sono ancora disponibili sufficienti evidenze in grado di garantire applicazioni cliniche riproducibili per le quali sono necessari studi clinici sempre più rigorosi e accurati, in mo- do da chiarire numerosi aspetti di questo affascinante settore della ricerca.

\section{Il PRP: the basics}

Il PRP è un concentrato autologo di piastrine in un piccolo volume di plasma $[4,5]$ con una concentrazione di piastrine superiore a 1 milione per millilitro di siero o cinque volte il valore basale [6], necessaria per stimolare efficacemente le cellule danneggiate alla guarigione $[7,8]$.

In realtà, a testimonianza dei numerosi dubbi e aspetti ancora oscuri sulla biologia di questa metodica terapeutica, uno studio recente di Fleming [9] ha mostrato come, invece, soltanto un numero di piastrine al livello basale possa migliorare i processi di guarigione.

Il razionale d'utilizzo del PRP, ad ogni modo, si basa sulla conoscenza ormai consolidata di alcuni processi biologici. Le piastrine sono la prima componente ematica a giungere sul sito della lesione, e hanno il potenziale di rilasciare fattori di crescita che giocano un ruolo decisivo nel modulare la risposta riparativa tissutale [10]. Questi fattori di crescita vengono rilasciati dai granuli piastrinici e la loro degranulazione può essere attivata utilizzando, in vitro, trombina o altri fattori di induzione ambientale $[11,12]$. La maggior parte di queste molecole può essere rilasciata nell'arco di 10 minuti circa, costituendo quindi una risposta estremamente 
rapida all'insulto esterno [13]. Alcuni di questi growth factors sono selettivamente regolati da proteine dei granuli sia per l'attivazione che per l'inibizione [14] e sono oggetto di studio i meccanismi endogeni o esogeni che possono attivare i granuli a secernere alte concentrazioni di fattori di crescita [15].

Se i progressi nel campo della ricerca di base hanno chiarito gran parte degli aspetti biologici fino a pochi anni fa sconosciuti, il problema è il passaggio alla ricerca clinica. A fronte di numerosi studi pubblicati, infatti, sono pochi gli ambiti nei quali vi sia un consenso unanime, come circa l'ottimale metodica di preparazione e composizione del PRP per ciascuna differente indicazione clinica [16]. Oltre a ciò, vi sono numerosi dubbi sul fatto che un singolo preparato possa trattare condizioni patologiche differenti. Molti fattori di crescita e citochine presenti nel PRP, infatti, agiscono dentro percorsi biologici opposti e alcuni hanno effetti benefici in determinate applicazioni cliniche e deleteri in altre. Mentre, ad esempio, il TGF1 può avere effetti benefici profibrotici sulla guarigione dei tendini e dei legamenti [17] è stato dimostrato un suo effetto negativo sulle lesioni muscolari [18]. La comprensione del ruolo di ciascun fattore di crescita nello sviluppo delle specifiche patologie faciliterà l'identificazione delle componenti efficaci per ciascuna indicazione e lo sviluppo di preparazioni "customizzate" adatte alle specifiche differenti indicazioni.

Allo stesso modo, vanno chiariti alcuni aspetti come la variabile presenza di leucociti all'interno del preparato. Il PRP a bassa concentrazione di leucociti è indicato come agente in grado di indurre la crescita cellulare stimolando i processi anabolici dei condrociti, mentre il PRP ad alta concentrazione di leucociti promuove i processi catabolici che coinvolgono varie citochine [19] e possono produrre numerosi effetti differenti [20].

Un ulteriore problema, infine, è l'estrema variabilità delle preparazioni esistenti. La concentrazione di fattori di crescita dentro le preparazioni può essere influenzata da fattori donatore-specifici e dal metodo di "processamento" e applicazione dei fattori.

La conoscenza delle capacità rigenerative in vitro del PRP ha comunque orientato numerosi ricercatori a esplorarne l'uso per il trattamento delle patologie ortopediche quali quella tendinea [21], muscolare [22], cartilaginea [23] e ossea [24].

\section{Applicazioni cliniche}

L'efficacia del gel piastrinico nello stimolare i processi riparativi ne ha reso possibile l'utilizzo già alla fine degli anni '90, in applicazioni cliniche come gli innesti autologhi e gli innesti con un misto di bone graft e sostituti ossei $[25,26]$, oltre a numerose applicazioni in chirurgia odontoiatrica e plastica ricostruttiva delle mucose. Dopo pochi anni, l'utilizzo clinico del PRP è stato esteso ad altre branche della medicina, tra cui l'ortopedia, ed esistono, in letteratura, numerosi report sull'utilizzo di PRP negli interventi riparativi in chirurgia del ginocchio [27-30], della spalla [31-33], della caviglia [34-37], della colonna vertebrale [38] e del gomito [39, 40]. Nonostante la mancanza di solide evidenze e l'incompleta comprensione dei meccanismi biologici alla base delle patologie muscolo scheletriche e, di riflesso, del funzionamento del PRP, sono numerosi i campi di applicazione di questo prodotto biologico per il trattamento conservativo di patologie articolari, ossee e muscolo-tendinee, sicuramente un quadro ancora non definito e non basato su evidenze solide, di indicazioni all'utilizzo di questa metodica.

\section{Ricostruzione del LCA}

La scienza di base e i modelli animali hanno dimostrato il rilascio di fattori di crescita che stimolano l'angiogenesi intorno al neolegamento, il rimodellamento e l'integrazione all'interfaccia osso/impianto [41, 42]. Sulla base degli studi clinici, inoltre, non sono state riscontrate differenze negli outcome in pazienti trattati con e senza PRP come supporto alla ricostruzione del LCA [43]. Il più significativo effetto clinico dell'utilizzo di PRP in supporto alla ricostruzione del LCA si è riscontrato nella sua applicazione al sito di prelievo del graft rotuleo, inducendo una risposta riparativa dei tessuti e un miglioramento degli outcome $[44,45]$.

\section{Ricostruzione della cuffia dei rotatori}

Numerosi studi hanno chiarito la capacità del PRP di incidere positivamente sulla rigenerazione del tessuto tendineo a livello cellulare inibendo la risposta infiammatoria, esercitando un'azione protettiva contro lo stress ossidativo che può indurre l'apoptosi cellulare e stimolando il rilascio di fattori di crescita che inducono angiogenesi e rigenerazione del tessuto tendineo $[46,47]$.

Il PRP è stato utilizzato come trattamento conservativo o di supporto alla chirurgia, mostrando buoni risultati nei casi di lesioni di dimensioni limitate e in acuto. Per quanto riguarda le lesioni massive i risultati sono stati peggiori, con un'insufficiente azione sui processi riparativi e una quota significativa di progressione della lesione $[48,49]$.

Una recente metanalisi non ha riscontrato differenze negli outcome e nella percentuale di recidiva della lesione fra pazienti trattati con e senza PRP durante la riparazione artroscopica della cuffia dei rotatori [50].

\section{Tendinopatie}

In virtù delle sue proprietà biologiche sul tessuto tendineo [51], il PRP ha trovato diverse applicazioni nel campo delle tendinopatie. 


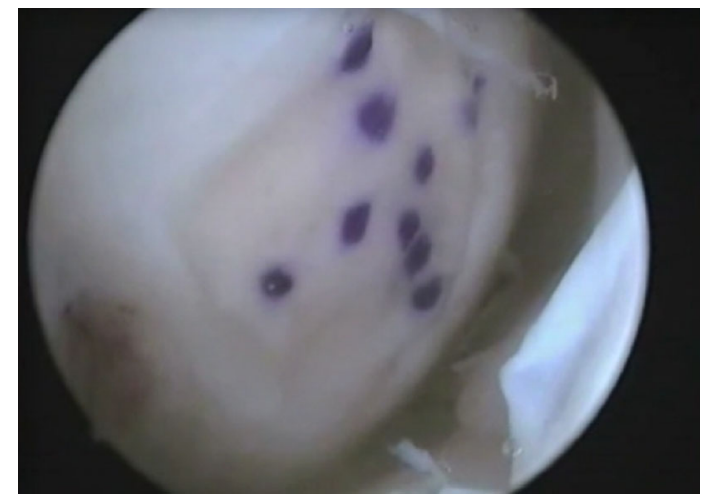

Fig. 1 - Lesione condrale del condilo femorale. Impianto di scaffold/PRP

Nel caso del tendine rotuleo, i dati sull'utilizzo del PRP sono inconsistenti [52] e nonostante, quindi, gli eventi avversi nell'utilizzo di questa metodica siano molto rari [53], l'efficacia del PRP nel trattamento di questa tenopatia non è ancora stata dimostrata.

Per quanto riguarda il tendine d'Achille, il PRP ha fornito risultati controversi, sia nella terapia conservativa che in quella chirurgica [54], mostrando maggiore efficacia sulle tendinopatie acute $[55,56]$. Crescente è l'attenzione all'utilizzo di questa metodica anche nel trattamento dell'epicondilite, in particolare nei casi refrattari alla terapia conservativa classica. La ricerca in questo campo ha registrato buoni risultati con follow-up a 6 settimane [57] o con follow-up più lungo a 24 settimane, osservando una diminuzione significativa del dolore e ripresa delle attività [58].

\section{Lesioni cartilaginee focali}

$\mathrm{Al}$ momento non esistono terapie in grado di restaurare con certezza il tessuto cartilagineo originale. Numerose tecniche riparative sono state introdotte nel corso degli anni, con buoni risultati clinici complessivi anche se non è possibile, al momento, ottenere nuova cartilagine ialina. Il PRP è stato proposto come supporto a queste terapie chirurgiche (Fig. 1) con risultati di difficile interpretazione.

La scienza di base e gli studi condotti su animali hanno mostrato risultati iniziali promettenti circa la capacità del PRP di regolare la proliferazione dei condrociti e la loro differenziazione, promuovere il rilascio di fattori di crescita limitando il processo infiammatorio e favorire la rigenerazione cartilaginea [59-63], documentata ai controlli RMN (Fig. 2). $\mathrm{Al}$ momento i risultati sono piuttosto frammentari e mancano outcome a lungo follow-up e comprendenti elementi clinici come dolore, score funzionale, dati clinici e radiografici comparabili.
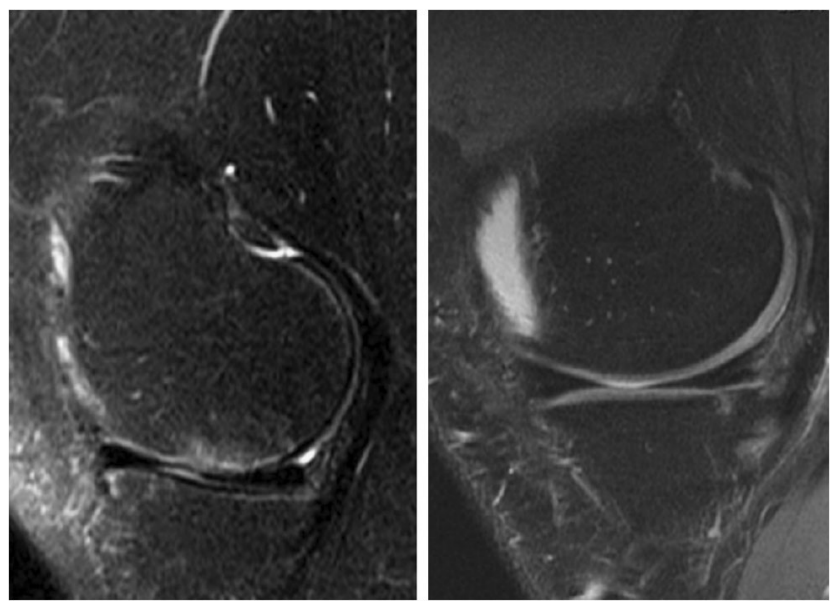

Fig. 2 - Lesione condrale del condilo femorale, prima e dopo trattamento con PRP a 6 mesi di follow-up

\section{Osteoartrosi}

La capacità del PRP di determinare una diminuzione della risposta infiammatoria e una risposta positiva in termini di riduzione del dolore e incremento del miglioramento funzionale [64] ne hanno fatto un candidato ideale alla terapia conservativa dell'osteoartrosi. Trial clinici che comparano acido ialuronico, PRP e placebo documentano la capacità del PRP di diminuire il dolore e migliorare la risposta funzionale in pazienti artrosici $[65,66]$. Gli effetti benefici del PRP in questo campo di applicazione appaiono molto più pronunciati e di durata maggiore nei pazienti più giovani e con deformità degenerative minori [67]. La revisione sistematica dei trial clinici pubblicati, dei case report e degli studi di coorte conferma risultati superiori con iniezioni locali di PRP rispetto all'acido ialuronico o al placebo [68-70].

$\mathrm{Al}$ momento, il dosaggio e la frequenza raccomandati per il trattamento con PRP o la tipologia di PRP da utilizzare per il trattamento intra-articolare rimane poco chiara [71], il che rende difficile formulare un giudizio complessivo. Il PRP ha, comunque, dimostrato risultati significativamente positivi sul dolore e il recupero funzionale e la terapia appare ben tollerata e priva di effetti collaterali significativi, mentre mancano elementi in grado di giungere a una standardizzazione delle modalità e del timing di applicazione clinica.

\section{Lesioni muscolari}

In questo ambito, forse maggiormente che in altri, i dati sono estremamente confusi, rendendo al momento impossibile formulare un giudizio definitivo in quanto manca un accordo comune sulle modalità di utilizzo del PRP e sulla raccolta degli outcome clinici [72-75]. Al momento non è possibile indicare in quali casi sia possibile utilizzare il PRP 


\begin{tabular}{ll}
\hline Consensus Statements on Platelet-rich Plasma \\
\hline No. & \multicolumn{1}{c}{ Statement } \\
\hline 1 & $\begin{array}{c}\text { An accepted nomenclature and classification system that } \\
\text { encompasses autologous blood/plasma products and categorizes } \\
\text { preparations in sufficient detail is required to facilitate comparison } \\
\text { across studies. Efforts should be made to involve academics, } \\
\text { clinicians, and industry representatives in this process to } \\
\text { encourage widespread adoption of the system. }\end{array}$ \\
The influence of donor variance and processing and delivery factors \\
on the composition of PRP must be established. \\
A validated assay of the efficacy of PRP should be established for \\
each clinical application. \\
The relationship between PRP composition and efficacy should be \\
established. \\
Minimum standards of reporting for all studies (preclinical and \\
clinical) evaluating PRP must be established to facilitate \\
communication and the interpretation and synthesis of scientific \\
investigations. These standards must include measured \\
characteristics of the PRP and factors relating to the donor, \\
processing, and delivery of the PRP.
\end{tabular}

Fig. 3 - Consensus statement sul PRP (da [76])

con la possibilità di ottenere effetti positivi e c'è bisogno di organizzare studi con maggiore rigorosità nella randomizzazione e nelle procedure di blinding per cui, al momento, il PRP non può essere considerato un'alternativa alle terapie classiche nelle lesioni muscolari ma un adiuvante che può supportare la terapia fisica e le altre strategie più consolidate per il trattamento di questi infortuni.

\section{Discussione}

L'utilizzo del PRP, nell'ambito delle terapie biologiche applicate all'ortopedia, resta un campo di ricerca affascinante e promettente, nonostante le controversie esistenti nella letteratura scientifica attuale.

Nel 2015, l'American Academy of Orthopaedic Surgeons (AAOS) ha tenuto un simposio per identificare i gap conoscitivi nell'ambito del trattamento biologico delle patologie ortopediche e stabilire direttive consensuali sulle direzioni che la ricerca in tale ambito dovrebbe prendere. Gli esperti che vi hanno partecipato hanno trovato comune accordo sul fatto che la via più efficace per ottenere una transizione significativa dalla ricerca di laboratorio alla pratica clinica è l'utilizzo di un approccio traslazionale basato su evidenze scientifiche e una maggiore integrazione fra ricercatori, legislatori e industria (Fig. 3).

Nonostante l'incertezza nei risultati, comunque, queste procedure stanno assumendo un ruolo sempre maggiore all'interno della pratica clinica, ponendo anche un problema relativo ai costi, in mancanza di un reale rapporto costi/benefici per modelli terapeutici che in virtù di queste incertezze non sono ancora basate su solide evidenze scientifiche.
Il problema dei costi è un elemento di riflessione condiviso in differenti ambiti di discussione scientifici e politici e, a tal proposito, la Fondazione GIMBE effettua periodicamente una ricerca dell'impatto degli sprechi sul Sistema Sanitario Nazionale, individuando al primo posto il "Sovra-utilizzo di interventi sanitari inefficaci e inappropriati", per un volume di 6,48 miliardi di euro. Tenere insieme i promettenti risultati della ricerca con le necessità di utilizzare procedure sicure ed evitare sprechi vuol dire considerare l'opzione delle terapie biologiche come frontiera da esplorare, procedendo a una standardizzazione dei criteri di selezione dei pazienti, delle misure di outcome, delle procedure cliniche e alla redazione di studi clinici randomizzati in cieco per una maggiore mole di dati statisticamente significativi a disposizione della ricerca.

Accanto a questo, sicuramente è auspicabile l'istituzione di registri regionali, coordinati dalle Università e dalla Società scientifiche, per l'avvio dei registri regionali, strumento essenziale per la raccolta dei dati e la loro analisi rigorosa, allo scopo di ottenere sempre maggiori informazioni significative dal punto di vista statistico e progredire nella conoscenza di queste metodiche, lavorando alla redazione di indicazioni specifiche, precise e standardizzate.

Conflitto Di interesse Gli autori A. Bove, B. Russo, F. Traficante, A. Barile e N. Orabona dichiarano di non aver alcun conflitto d'interesse.

CONSENSO INFORMATO E CONFORMITÀ AGLI STANDARD ETICI Tutte le procedure descritte nello studio e che hanno coinvolto essere umani sono state attuate in conformità alle norme etiche stabilite dalla dichiarazione di Helsinki del 1975 e successive modifiche. Il consenso informato è stato ottenuto da tutti i pazienti inclusi nello studio.

Human ANd ANimal Rights L'articolo non contiene alcuno studio eseguito su esseri umani e su animali da parte degli autori.

Open ACCESS This article is distributed under the terms of the Creative Commons Attribution 4.0 International License (http://creative commons.org/licenses/by/4.0/), which permits unrestricted use, distribution, and reproduction in any medium, provided you give appropriate credit to the original author(s) and the source, provide a link to the Creative Commons license, and indicate if changes were made.

\section{Bibliografia}

1. Sugaya K (2003) Potential use of stem cells in neurodegenerative diseases. Int Rev Cytol 228:1-30

2. Anz AW, Hackel JG, Nilssen EC, Andrews JR (2014) Application of biologics in the treatment of the rotator cuff, meniscus, cartilage and osteoarthritis. J Am Acad Orthop Surg 22(2):68-79

3. Gobbi A, Bathan I (2009) Biological approaches for cartilage repair. J Knee Surg 22(1):36-44

4. Marx RE (2004) Platelet-rich plasma: evidence to support its use. J Oral Maxillofac Surg 62:489-496

5. Zhu Y, Yuan M, Meng HY et al (2013) Basic science and clinical application of platelet-rich plasma for cartilage defects and osteoarthritis: a review. Osteoarthr Cartil 21(11):1627-1637 
6. Dhillon RS, Schwarz EM, Maloney MD (2012) Platelet-rich plasma therapy-future or trend? Arthritis Res Ther 14(4):219

7. Marx RE (2004) Platelet-rich plasma: what is PRP and what is not PRP? Implant Dent 10(4):225-228

8. Rughetti A, Giusti I, D’Ascenzo S et al (2008) Platelet-gel released supernatant modulates the angiogenic capability of human endothelial cells. Blood Transfus 6(1):12-17

9. Fleming BC, Proffen BL, Vavken P et al (2015) Increased platelet concentration does not improve function algraft healing in bio-enhanced ACL reconstruction. Knee Surg Sports Traumatol Arthrosc 23(4):1161-1170

10. Creaney L, Hamilton B (2008) Growth factor delivery methods in the management of sports injuries: the state of play. Br J Sports Med 42(5):314-320

11. Alsousou J, Thompson M, Hulley P et al (2009) The biology of platelet-rich plasma and its application in trauma and orthopaedic surgery: a review of the literature. J Bone Jt Surg, Br 91:987-996

12. Mishra A, Woodall J Jr, Vieira A (2009) Treatment of tendon and muscle platelet-rich plasma. Clin Sports Med 28:113-125

13. Marx RE, Carlson ER, Eichstaedt RM et al (1998) Platelet rich plasma: growth factor enhancement for bone grafts. Oral Surg Oral Med Oral Pathol Oral Radiol Endo 85(6):638-646

14. Italiano JE Jr, Richardson JL, Patel-Hett S et al (2008) Angiogenesis is regulated by a novel mechanism: pro- and antiangiogenetic proteins are organized into separate platelet alpha granules and differentially released. Blood 111(3):1227-1233

15. Foster TE, Puskas BL, Mandelbaum BR et al (2009) Platelet-rich plasma: from basic science to clinical applications. Am J Sports Med 37(11):2259-2272

16. Murray JR, La Prade RF, Musahl V et al (2016) Biologic treatments for sports injuries II think thank-current concepts, future research and barriers to advancement part 2: rotator cuff. Orthop J Sports Med 4(3):23259671

17. Beye JA, Hart DA, Bray RC et al (2008) Injury-induced changes in mRNA levels differ widely between anterior cruciate ligament and medial collateral ligament. Am J Sports Med 36(7):1337-1346

18. Huard J, Li Y, Fu FH (2002) Muscle injuries and repair: current trends in research. J Bone Jt Surg, Am 84(5):822-832

19. Cavallo C, Filardo G, Mariani E et al (2014) Comparison of platelet-rich plasma formulations for cartilage healing: an in vitro study. J Bone Jt Surg, Am 96(5):423-429

20. Filardo G, Kon E, Pereira Ruiz MT et al (2012) Platelet-rich plasma intra-articular injections for cartilage degeneration and osteoarthritis: single versus double-spinning approach. Knee Surg Sports Traumatol Arthrosc 20(10):2082-2091

21. Andia I, Sanchez M, Maffulli N (2010) Tendon healing and platelet-rich plasma therapies. Expert Opin Biol Ther 10(10):1415-1426

22. Hamid MS, Mohamed Ali MR, Yusof A et al (2014) Plateletrich plasma injections for the treatment of hamstring injuries: a randomized controlled trial. Am J Sports Med 42(10):2410-2418

23. Kon E, Mandelbaum B, Buda R et al (2011) Platelet-rich plasma intra-articular injection versus hyaluronic acid viscosupplementation as treatments for cartilage pathology: from early degeneration to osteoarthritis. Arthroscopy 27(11):1490-1501

24. Mazzocca A, Mc Carthy MB, Chowaniec DM et al (2012) The positive effects of different platelet—rich plasma methods on human muscle, bone and tendon cells. Am J Sports Med 40(8):1742-1749

25. Weibrich G, Gnoth SH, Otto M et al (2002) Growth stimulation of human osteoblast-like cells by thrombocyte concentrates in vitro. Mund Kiefer Geschtschir 6:168

26. Garg AK (2000) The use of platelet rich plasma to enhance the success of bone grafts around dental implants. Dent Implantol Update $11: 17$
27. Ekdahl M, Wang JH, Ronga M, Fu FH (2008) Graft healing in anterior cruciate ligament reconstruction. Knee Surg Sports Traumatol Arthosc 16(10):935-947

28. Chen CH (2009) Graft healing in anterior cruciate ligament reconstruction. Sports Med Arthrosc Rehabil Ther Technol $1(1): 21$

29. Silva A, Sampaio R (2009) Anatomic ACL reconstruction: does the platelet-rich plasma accelerate tendon healing? Knee Surg Sports Traumatol Arthrosc 17(6):676-682

30. Andriolo L, Di Matteo B, Kon E et al (2015) PRP augmentation for ACL reconstruction. BioMed Res Int 2015:371746

31. Scarpone M, Rabago D, Snell E et al (2013) Effectiveness of platelet-rich plasma injection for rotator cuff tendinopathy: a prospective open-label study. Glob Adv Health Med 2(2):26-31

32. Ersen A, Demirhan M, Atalar AC et al (2014) Platelet rich plasma for enhancing surgical rotator cuff repair: evaluation and comparison of two application methods in a rat model. Arch Orthop Trauma Surg 134(3):405-411

33. Zhao JG (2015) Platelet-rich plasma in arthroscopic rotator cuff repair. Arthroscopy 31(4):597-598

34. Creaney L (2010) Platelet-rich plasma for treatment of Achilles tendinopathy. JAMA 303(17):1696

35. de Vos RJ, Weir A, van Schie HT et al (2010) Platelet-rich plasma injection for chronic Achilles tendinopathy: a randomized controlled trial. JAMA 303(2):144-149

36. de Jonge S, de Vos RJ, Weir A et al (2011) One-year follow up of platelet-rich plasma treatment in chronic Achilles tendinopathy: a double blind randomized placebo-controlled trial. Am J Sports Med 39(8):1623-1629

37. Aspenberger P (2013) Platelet concentrates and Achilles tendon healing. J Orthop Res 31(9):1500

38. Hartmann EK, Heintel T, Morrison RH, Weckbach A (2010) Influence of platelet-rich plasma on the anterior fusion in spinal injuries: a qualitative and quantitative analysis using computer tomography. Arch Orthop Trauma Surg 130(7):909-914

39. Thanasas C, Papadimitriou G, Charalambidis C et al (2011) Platelet-rich plasma versus autologous whole blood for the treatment of chronic lateral elbow epicondylitis: a randomized controlled trial. Am J Sports Med 39(10):2130-2314

40. Mishra AK, Skrepnik NV, Edwards SG et al (2014) Efficacy of platelet-rich plasma for chronic tennis elbow: a double-blind, prospective, multicenter, randomized trial of 230 patients. Am J Sports Med 42(2):463-471

41. Anderson K, Seneviratne AM, Izawa K et al (2001) Augmentation of tendon healing in an intraarticular bone tunnel with use of a bone growth factor. Am J Sports Med 29:689-698

42. Yamazaki S, Yasuda K, Tomita F et al (2005) The effect of transforming growth factor-beta1 on intra osseous healing of flexor tendon autograft replacement of anterior cruciate ligament in dogs. Arthroscopy 21:1034-1041

43. Vavken P, Sadoghi P, Murray MM (2011) The effect of platelet concentrates on graft maturation and graft-bone interface healing in anterior cruciate ligament reconstruction in human patients: a systematic review of controlled trials. Arthroscopy 27:1573-1583

44. Cervellin M, de Girolamo L, Bait C et al (2012) Autologous platelet-rich plasma gel to reduce donor-site morbidity after patellar tendon graft harvesting for anterior cruciate ligament reconstruction. A randomized, controlled clinical study. Knee Surg Sports Traumatol Arthrosc 20:114-120

45. de Almeida AM, Demange K, Sobrado MF et al (2012) Patellar tendon healing with platelet—rich plasma: a prospective randomized controlled trial. Am J Sports Med 40:1282-1288 
46. Jo CH, Kim JE, Yoon KS, Shin S (2012) Platelet-rich plasma stimulates cell proliferation and enhances matrix gene expression and synthesis in tenocytes from human rotator cuff tendons with degenerative tears. Am J Sports Med 40:1035-1045

47. Namazi H (2011) Rotator cuff repair healing influenced by platelet-rich plasma construct augmentation: a novel molecular mechanism. Arthroscopy 27:1456

48. Bergeson AG, Tashijan RZ, Greis PE et al (2012) Effects of plateletrich fibrin matrix on repair integrity of at-risk rotator cuff tears. Am J Sports Med 40:286-293

49. Castricini R, Longo UG, De Benedetto M et al (2011) Plateletrich plasma augmentation for arthroscopic rotator cuff repair: a randomized controlled trial. Am J Sports Med 39:258-265

50. Warth RJ, Dornan GJ, James EW et al (2015) Clinical and structural outcomes after arthroscopic repair of full-thickness rotator cuff tears with and without platelet-rich product supplementation. A metanalysis and meta-regression. Arthroscopy 31:306-320

51. Zhang J, Wang JH (2010) Platelet-rich plasma releasate promotes differentiation of tendon stem cells into activate nocytes. Am J Sports Med 38:2477-2486

52. Liddle AD, Rodriguez-Merchan EC (2015) Platelet-rich plasma in the treatment of patellar tendinopathy: a systematic review. Am J Sports Med 43:2583-2590

53. Bowman KF, Muller B, Middleton K et al (2013) Progression of patellar tendinitis following treatment with platelet-rich plasma: case reports. Knee Surg Sports Traumatol Arthrosc 21:2035-2039

54. Schepull T, Kvist J, Norrman H et al (2011) Autologous platelet shave no effect on the healing of human Achilles tendon ruptures: a randomized, single blind study. Am J Sports Med 39:38-47

55. de Jonge S, de Vos RJ (2011) One-year follow up of platelet-rich plasma treatment in chronic Achilles tendinopathy: a double blind randomized placebo-controlled trial. Am J Sports Med 39:16231629

56. de Vos RJ, Weir A, Tol JL et al (2011) No effects of PRP on ultrasonographic tendon structure and neovascularization in chronic midportion Achilles tendinopathy. Br J Sports Med 45:387-392

57. Thanasas C, Papadimitriou G, Charalambidis C et al (2011) Platelet-rich plasma versus autologous wholeblood for the treatment of chronic lateral elbow epicondylitis: a randomized controlled trial. Am J Sports Med 39:2130-2134

58. Mishra AK, Skrepnik NV, Edwards SG et al (2014) Efficacy of platelet-rich plasma for chronic tennis elbow: a double-blind, prospective, multi-center, randomized controlled trial of 230 patients. Am J Sports Med 42:463-471

59. Kisiday JD, McIlwraith CW, Rodkey WG et al (2012) Effects of platelet-rich plasma composition on anabolic and catabolic activities in equine cartilage and meniscal explants. Cartilage 3:245-254

60. Lee CH, Cool JL, Mendelson A et al (2010) Regeneration of the articular surface of the rabbit synovial joint by cell homing: a proof of concept study. Lancet 376:440-448

61. Petrera M, de Croos JN, Iu J et al (2013) Supplementation with platelet-rich plasma improves the in vitro formation of tissue-engineered cartilage with enhanced mechanical properties. Arthroscopy 29:1685-1692

62. Smyth NA, Murawski CD, Fortier LA et al (2013) Platelet-rich plasma in the pathologic processes of cartilage: a review of basic science evidence. Arthroscopy 29:1399-1409

63. Xie X, Zhang C, Tuan RS (2014) Biology of platelet-rich plasma and its clinical application in cartilage repair. Arthritis Res Ther $16: 204$

64. van Buul GM, Koevoet WL, Kops N et al (2011) Platelet-rich plasma releseate inhibits inflammatory processes in osteoarthritic chondrocytes. Am J Sports Med 39:2362-2370

65. Cerza F, Carnì S, Carcangiu A et al (2012) Comparison between hyaluronic acid and platelet-rich plasma, intra-articular infiltration in the treatment of gonarthrosis. Am J Sports Med 27:1490-1501

66. Kon E, Mendelbaum B, Buda R et al (2011) Platelet-rich plasma intra articular injection versus hyaluronic acid viscosupplementation as treatments for cartilage pathology: from early degeneration to osteoarthritis. Arthroscopy 27:1490-1501

67. Filardo G, Kon E, Pereira Ruiz MT et al (2012) Platelet-rich plasma intra-articular injections for cartilage degeneration and osteoarthritis: single versus double-spinning approach. Knee Surg Sports Traumatol Arthrosc 20:2082-2091

68. Xie X, Zhang C, Tuan RS (2014) Biology of platelet-rich plasma and its clinical application in cartilage repair. Arthritis Res Ther $16: 204$

69. Campbell KA, Saltzmann BM, Mascarenhas R et al (2015) Does intra-articular platelet-rich plasma injection provide clinically superior outcomes compared with other therapies in the treatment of knee osteoarthritis? A systematic review of overlapping meta-analyses. Arthroscopy 31:2213-2221

70. Gobbi A, Lad D, Karnatzikos G (2015) The effects of repeated intra-articular PRP injections on clinical outcomes of early osteoarthritis of the knee. Knee Surg Sports Traumatol Arthrosc 23:2170-2177

71. Sundman EA, Cole BJ, Karas V et al (2014) The antinflammatory and matrix restorative mechanism of platelet-rich plasma in osteoarthritis. Am J Sports Med 42(1):35-41

72. Hammond JW, Hinton RY, Curl LA et al (2010) Use of autologous platelet-rich plasma to treat muscle strain injuries. Am J Sports Med 37(6):1135-1142

73. Harris NL, von Huffer WE, Stade E et al (2012) The effect of platelet-rich plasma on normal soft tissues in the rabbit. J Bone Jt Surg, Am 94(9):786-793

74. Guillodo Y, Madouas G, Simon T et al (2015) Platelet-rich plasma (PRP) treatment of sports-related severe acute hamstrings injuries. Muscle Ligaments Tendons 5(4):284-288

75. Reurink G, Goudsward GJ, Moen MH et al (2014) Dutch hamstring injection therapy (HIT) study investigators. N Engl J Med 370(26):2546-2547

76. La Prade RF, Dragoo JL, Koh JL et al (2016) AAOOS Research Symposium Updates and consensus: biologic treatment for Orthopaedic injuries. J Am Acad Orthop Surg 24:e62-e78 